University of Nebraska - Lincoln

DigitalCommons@University of Nebraska - Lincoln

July 2000

\title{
Carbon Nanotube-Based Nonvolatile Random Access Memory for Molecular Computing
}

Thomas Rueckes

Harvard University, Cambridge, MA

Kyoungha Kim

Harvard University, Cambridge, MA

Ernesto Joselevich

Harvard University, Cambridge, MA

Greg Y. Tseng

Harvard University, Cambridge, MA

Chin Li Cheung

University of Nebraska at Lincoln, ccheung2@unl.edu

See next page for additional authors

Follow this and additional works at: https://digitalcommons.unl.edu/chemistrycheung

Part of the Chemistry Commons

Rueckes, Thomas; Kim, Kyoungha; Joselevich, Ernesto; Tseng, Greg Y.; Cheung, Chin Li; and Lieber, Charles M., "Carbon Nanotube-Based Nonvolatile Random Access Memory for Molecular Computing" (2000). Barry Chin Li Cheung Publications. 9.

https://digitalcommons.unl.edu/chemistrycheung/9

This Article is brought to you for free and open access by the Published Research - Department of Chemistry at DigitalCommons@University of Nebraska - Lincoln. It has been accepted for inclusion in Barry Chin Li Cheung Publications by an authorized administrator of DigitalCommons@University of Nebraska - Lincoln. 


\section{Authors}

Thomas Rueckes, Kyoungha Kim, Ernesto Joselevich, Greg Y. Tseng, Chin Li Cheung, and Charles M. Lieber 


\title{
Carbon Nanotube-Based Nonvolatile Random Access Memory for Molecular Computing
}

\author{
Thomas Rueckes, ${ }^{1}$ Kyoungha Kim, ${ }^{2}$ Ernesto Joselevich, ${ }^{1}$ Greg Y. Tseng, ${ }^{1}$ \\ Chin-Li Cheung, ${ }^{1}$ and Charles M. Lieber ${ }^{1,2, *}$ \\ ${ }^{1}$ Department of Chemistry and Chemical Biology, Harvard University, Cambridge, MA 02138, USA \\ 2 Division of Engineering and Applied Sciences, Harvard University, Cambridge, MA 02138, USA. \\ * Corresponding author; email: cml@cmliris.harvard.edu
}

A concept for molecular electronics exploiting carbon nanotubes as both molecular device elements and molecular wires for reading and writing information was developed. Each device element is based on a suspended, crossed nanotube geometry that leads to bistable, electrostatically switchable ON/OFF states. The device elements are naturally addressable in large arrays by the carbon nanotube molecular wires making up the devices. These reversible, bistable device elements could be used to construct nonvolatile random access memory and logic function tables at an integration level approaching $10^{12}$ elements per square centimeter and an element operation frequency in excess of 100 gigahertz. The viability of this concept is demonstrated by detailed calculations and by the experimental realization of a reversible, bistable nanotube-based bit.

In the past several decades, there has been a nearly constant exponential growth in the capabilities of silicon-based microelectronics (1). However, it is unlikely that these advances will continue much into the new millennium, because fundamental physical limitations, which prevent current designs from functioning reliably at the nanometer scale, will be reached while at the same time exponentially rising fabrication costs will make it prohibitive to raise integration levels. Molecular electronics $(2,3)$ can in principle overcome these limitations of silicon technology, because it is possible to have single-molecule devices that are organized cheaply in parallel by self-assembly. Much effort in this area has been focused on organic molecules as device elements, with very recent demonstrations of irreversible switches (4) and large negative differential resistances (5) for ensembles of molecules sandwiched between metal electrodes. The connection of molecular switching elements to the molecular wires that will be required for high-density integration and the function of such structures remains a substantial challenge.

Nanometer-diameter single-walled carbon nanotubes (SWNTs) exhibit unique electronic, mechanical, and chemical properties that make them attractive building blocks for molecular electronics $(6,7)$. Depending on diameter and helicity, SWNTs behave as onedimensional metals or as semiconductors (8), which, by virtue of their great mechanical toughness and chemical inertness, represent ideal materials for creating reliable, highdensity input/output (I/O) wire arrays. However, viable strategies for introducing molecular-scale device functionality into such $\mathrm{I} / \mathrm{O}$ lines have not been established. SWNTs have been used to make low-temperature singleelectron (9) and room temperature field effect
(10) transistors. Smaller devices based on intratube junctions have been proposed (11) and observed recently in experiments (12), although no approaches yet exist for either the controlled synthesis of nanotube junctions or the integration of many addressable junctions as needed for molecular-scale computing.

Our concept for integrated molecular electronics differs substantially from previous efforts (2-5), because it exploits a suspended SWNT crossbar array for both I/ $\mathrm{O}$ and switchable, bistable device elements with well-defined OFF and ON states (Figure 1). This crossbar consists of a set of parallel SWNTs or nanowires on a substrate and a set of perpendicular SWNTs that are suspended on a periodic array of supports (Figure 1A). Each cross point in this structure corresponds to a device element with a SWNT suspended above a perpendicular nanoscale wire. Qualitatively, bistability can be envisioned as arising from the interplay of the elastic energy, which produces a potential energy minimum at finite separation (when the upper nanotube is freely suspended), and the attractive van der Waals (vdW) energy, which creates a second energy minimum when the suspended SWNT is deflected into contact with the lower nanotube. These two minima correspond to welldefined OFF and ON states, respectively; that is, the separated upper-to-lower nanotube junction resistance will be very high, whereas the contact junction resistance will be orders of magnitude lower. A device element could be switched between these well-defined OFF and ON states by transiently charging the nanotubes to produce attractive or repulsive electrostatic forces. On the basis of this switching mode, we can characterize the elements as nano- or molecular-scale electromechanical devices.

In the integrated system, electrical con- tacts are made only at one end of each of the lower and upper sets of nanoscale wires in the crossbar array, and thus, many device elements can be addressed from a limited number of contacts (Figure 1B). At each cross point $(n, m)$ in the array, the suspended (upper) SWNT can exist in either the separated OFF state or the ON state in contact with the perpendicular nanotube on the substrate (lower SWNT). The ON/OFF information at an $(n, m)$ element thus can be read easily by measuring the resistance of the junction and, moreover, can be switched between OFF and ON states by applying voltage pulses at electrodes $n$ and $m$. This approach suggests highly integrated, fast, and macroscopically addressable nonvolatile random access memory (RAM) structure that could overcome the fundamental limitations of semiconductor RAM in size, speed, and cost.

To quantify the bistability and switching behavior of the proposed device element, we calculated the total energy $E_{\mathrm{T}}$

$$
E_{\mathrm{T}}=E_{\mathrm{vdw}}+E_{\text {elas }}+E_{\text {elec }}
$$

where $E_{\mathrm{vdw}}$ is the vdW energy, $E_{\text {elas }}$ is the elastic energy, and $E_{\text {elec }}$ is the electrostatic energy for the device. The first two terms in Equation 1, which define the static potential, were evaluated to assess the range of parameters that yield bistable devices. The vdW interaction between nanotubes was calculated by pairwise summation of a Lennard-Jones potential that has been previously shown to provide good agreement with experiments for nanotube systems $(13,14)$. The elastic contribution to the total energy was determined with a beam mechanics model

$$
E_{\text {elas }}=\frac{6\left(k^{3} B\right)^{1 / 4}}{\sqrt{2}\left[3+3 \beta L+3(\beta L)^{2}+(\beta L)^{3}\right]}(\delta z)^{2}
$$

where $B$ is the product of the nanotube elastic 
modulus and geometric moment of inertia, $k$ is the elastic modulus of the support, $L$ is the length of the suspended nanotube, $\beta=1 / \sqrt{2}$ $(k / B)^{1 / 4}$, and $\delta z$ is the displacement of the suspended tube from its unstrained position (15). Our calculations show that the proposed SWNT device structure will exhibit bistability for a broad range of parameters (Figure 2). The 20-nm device in Figure 2A exhibits $\mathrm{ON}$ and $\mathrm{OFF}$ states that are stable at room temperature (i.e., barrier $\gg 10 k_{\mathrm{B}} T$; $k_{\mathrm{B}}$ is the Boltzmann constant and $T$ is temperature) for initial separations ranging from 1.0 to $2.2 \mathrm{~nm}$. The calculated structures of the SWNT device element in the OFF and ON states for an initial separation of $2 \mathrm{~nm}$ (Figure 2B) highlight the relatively minor distortion of the upper SWNT in the ON state (this nanotube does not buckle or kink when deformed), even when the initial separation is near the upper limit for bistability and deformation is at a maximum. These calculations also show that the potential is bistable for a wide range of device sizes when the upper nanotube is supported on either hard (Figure 2C) or soft organic (Figure 2D) materials. The minimum bistable device size for a hard support
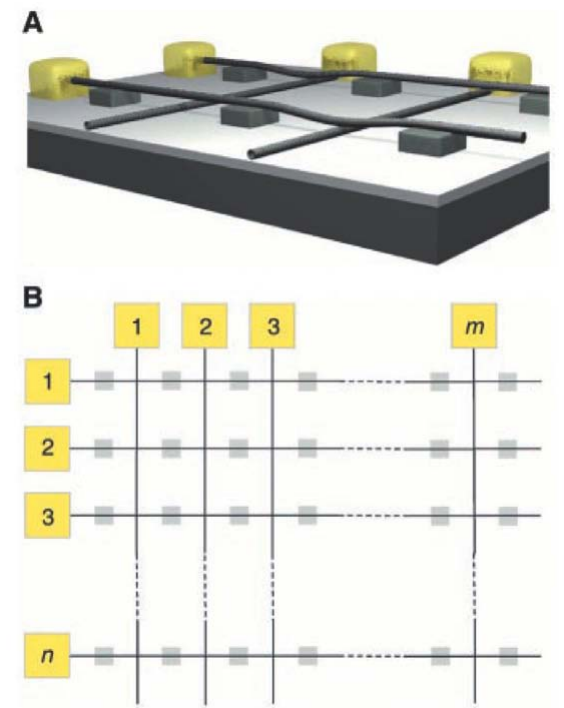

Figure 1. Suspended nanotube device architecture. (A) Three-dimensional view of a suspended crossbar array showing four junctions with two elements in the ON (contact) state and two elements in the OFF (separated) state. The substrate consists of a conducting layer [e.g., highly doped silicon (dark gray)] that terminates in a thin dielectric layer [e.g., $\mathrm{SiO}_{2}$ (light gray)]. The lower nanotubes are supported directly on the dielectric film, whereas the upper nanotubes are suspended by periodic inorganic or organic supports (gray blocks). Each nanotube is contacted by a metal electrode (yellow blocks). (B) Top view of an $n$ by $m$ device array. The nanotubes in this view are represented by black crossing lines, and the support blocks for the suspended SWNTs are indicated by light gray squares. The electrodes used to address the nanotubes are indicated by yellow squares. such as silicon is $<10 \mathrm{~nm}$, and softer organic supports enable bistability for devices that are $<5 \mathrm{~nm}$. Both types of materials could be envisioned for device fabrication.

There are several important points that can be drawn from these calculations. First, there is a wide range of parameters that yield a bistable potential for the proposed device configuration. The robustness of the ON/OFF states strongly suggests that this architecture will be tolerant of variations in structure that inevitably arise during fabrication by, for example, self-assembly. Second, the differences in separation between nanotubes in the $\mathrm{ON}$ and OFF states will produce large differences in resistance [i.e., $I \sim \exp (-k d)$, where $I$ is the current, $k$ is a decay constant on the order of $2 \AA^{-1}$, and $d$ is the tube-tube separation in angstroms] and thus should enable reliable reading of the $\mathrm{ON}$ and $\mathrm{OFF}$ states independent of variations in cross-contact resistance. Third, the range of mechanical strains required to achieve bistability in Figure $2 \mathrm{~A}, 0.22$ to $1.7 \%$, is well below the elastic limit of at least $6 \%$ [determined computationally (16) and experimentally (17) for SWNTs], and the average bending angle in the ON state is about half the angle required to buckle nanotubes. Hence, these device elements should be robust as required for a reliable molecular-scale computer (18), and the nanotube electronic properties should not be substantially affected by deformation to the ON state. The range of strains does exceed, however, the failure limit of most other materials and thus suggests that SWNTs are crucial for the suspended molecular wire. Lastly, these results can be used to address the possible issue of mechanical cross talk in device arrays. Comparison of the calculated strain energies to values of the nanotube-surface interaction (14) and friction suggests that (i) the lower nanotube will remain fixed on the substrate and (ii) the suspended nanotubes will not lift off or slip on supports on the order of $10 \mathrm{~nm}$ when the suspended tube is deflected to the ON state. The interaction with the support could also be enhanced through chemical modification. These results indicate that mechanical cross talk will not be a problem for our architecture in densely integrated crossed nanotube arrays.

Electromechanical switching of the suspended nanotube devices between $\mathrm{ON}$ and
A

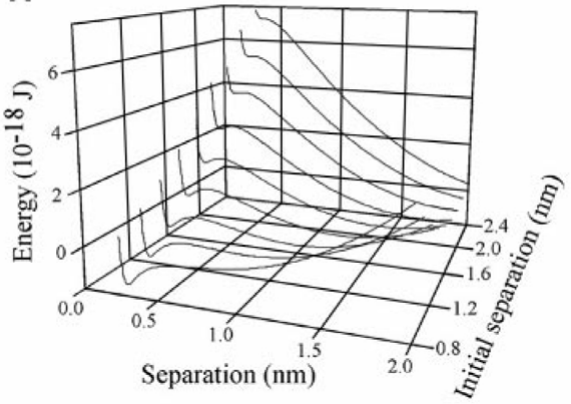

C

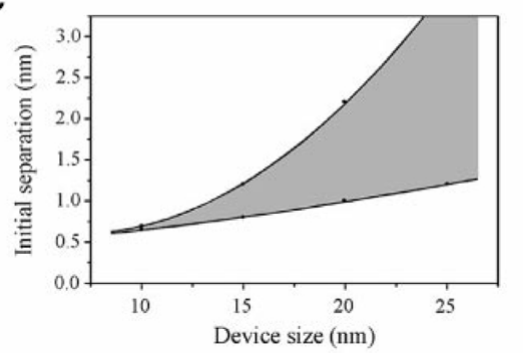

B

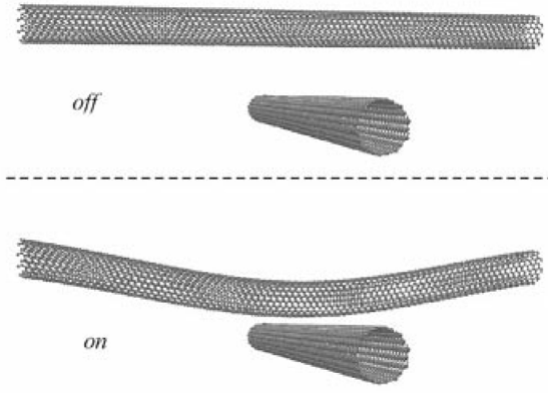

D

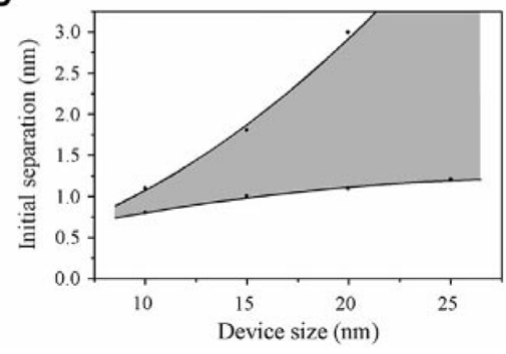

Figure 2. Bistable nanotube device potential. (A) Plots of energy, $E_{\mathrm{T}}=E_{\mathrm{vdW}}+E_{\mathrm{elas}}$, for a single 20-nm device as a function of separation at the cross point. The series of curves correspond to initial separations of $0.8,1.0,1.2,1.4,1.6,1.8,2.0,2.2$, and $2.4 \mathrm{~nm}$, with two well-defined minima observed for initial separations of 1.0 to $2.0 \mathrm{~nm}$. These mimima correspond to the crossing nanotubes being separated and in vdW contact. (B) Calculated structures of the 20-nm $(10,10)$ SWNT device element in the OFF (top) and ON (bottom) states. The initial separation for this calculation was $2.0 \mathrm{~nm}$; the silicon support structures (elastic modulus of $168 \mathrm{GPa}$ ) are not shown for clarity. (C and D) Plots of the bistability range for the crossed nanotube elements as a function of device size. The suspended nanotube is supported on silicon (C) or on an organic layer (D) with an elastic modulus of $12 \mathrm{GPa}$ (29). In both plots, the range of initial separations yielding bistable devices is gray. In general, the bistable region increases with device size, and the softer organic support yields a larger range of bistability, especially in the smallest structures. The calculations were carried out for $(10,10)$ SWNTs with an elastic modulus of $1 \mathrm{TPa}$ and Lennard-Jones parameters of $C_{6}=32.00 \times 10^{-60} \mathrm{erg} \cdot \mathrm{cm}^{6}$ and $C_{12}=55.77 \times 10^{-105} \mathrm{erg} \cdot \mathrm{cm}^{12}$. 
OFF states has been assessed by evaluating the voltage-dependent contribution of the electrostatic energy to the total energy. In this calculation, we used the boundary element method to numerically solve the Laplace equation for the complex three-dimensional (3D) geometry of the crossed nanotube device (19). Calculations of $E_{\mathrm{T}}$ for switching a 20-nm device ON and OFF (Figure 3) demonstrate that it is possible to change reversibly between the ON/OFF states by using moderate voltages, which do not exceed the threshold field for nanotube failure (20). The switching voltages vary, depending on the specific device geometry (i.e., shape of the static potential), and thus could be further optimized. For example, by using a thinner dielectric layer (that is, 4- versus 20-nm $\mathrm{SiO}_{2}$ ) the $\mathrm{ON}$ and OFF switching thresholds

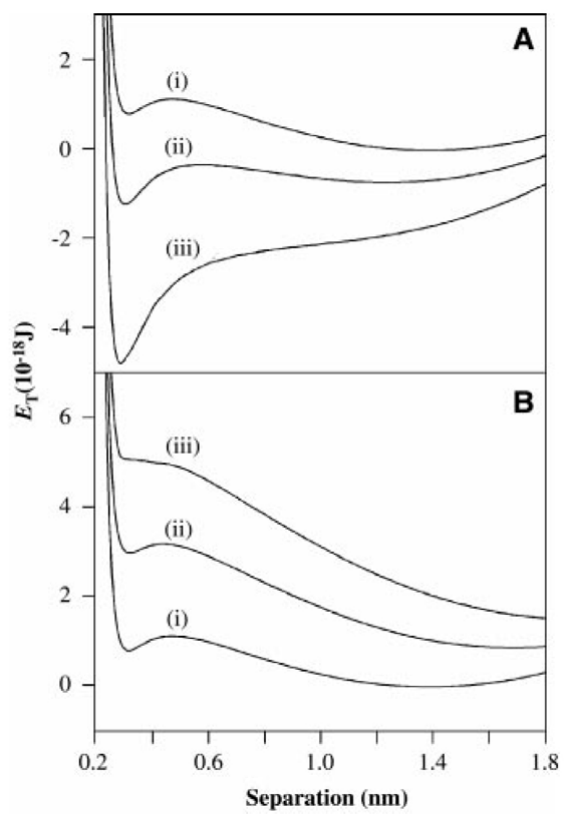

Figure 3. Electrostatic switching of the nanotube device. Plots of the energy, $E_{\mathrm{T}}=E_{\mathrm{vdW}}+E_{\text {elas }}+E_{\text {electro, }}$ as a function of separation at the cross point for (A) switching ON and (B) switching OFF. In (A), plots (i), (ii), and (iii) correspond to $E_{T}$ for $V_{1}=V_{2}=0 \mathrm{~V}$ and $V_{1}=+3 \vee, V_{2}=-3 \vee$ and $V_{1}=+4.5$, and $V_{2}=-4.5 \mathrm{~V}$, respectively, where $V_{1}$ and $V_{2}$ are the potentials applied to the two crossing nanotubes. In (B), (i), (ii), and (iii) correspond to $V_{1}=V_{2}=0 \mathrm{~V}, V_{1}=V_{2}=+15 \mathrm{~V}$, and $V_{1}=V_{2}=+20 \mathrm{~V}$, respectively. These potentials are applied with respect to the conducting ground plane (e.g., Figure 1A). The minimum magnitudes of the voltages required for switching ON and OFF are 4.5 and $20 \mathrm{~V}$, respectively. The electrostatic energy was calculated by numerically solving the Laplace equation using the boundary element method with 3600 elements for a $20-\mathrm{nm}$ device supported on silicon with a 1.4-nm initial separation. The calculated electrostatic potential was checked carefully to see that it satisfied the boundary conditions and asymptotic behavior. would be reduced from 4.5 and $20 \mathrm{~V}$ to 3 and $5 \mathrm{~V}$, respectively. The calculations also show that the electrostatic forces between adjacent nanotubes are insufficient to distort an array of elements, even at a $10-\mathrm{nm}$ device scale, because most of the electrostatic interaction is localized in the small crossing region of the individual elements.

Our proposed electromechanical device elements differ in important ways from previous fullerene (21) and nanotube (18) electromechanical systems. In the fullerene case, scanning tunneling microscopy (STM) measurements showed that the conductance of individual $\mathrm{C}_{60}$ clusters varied as a function of deformation by the STM tip. The variation in conductance can be associated with $\mathrm{ON}$ and OFF states, although the "device" can neither store information, because the change is reversible, nor can any scheme integrate many elements as required for computing. The nanotube nanotweezers (18), which consist of two nanotubes with freely suspended ends, do exhibit electrostatically switchable OFF (open) and ON (closed) states but cannot be readily integrated into a parallel system required for computing.

The bistable and reversible nanotube device elements can be used both as nonvolatile RAM and as configurable logic tables and thus could serve as the key building blocks for a molecular-scale computer. The potential of a system based on these nanotube device elements is substantial. First, it will be possible to achieve integration levels as high as $1 \times 10^{12}$ elements per square centimeter using 5-nm device elements and 5-nm supports while maintaining the addressability of many devices through the long $(\sim 10-\mu \mathrm{m})$ SWNT wires. In our architecture, interconnects to the outside are needed only at the ends of these long nanotubes, and thus, one interconnect can be used to address many individual junction elements. We also stress that each element can store a nonvolatile bit, whereas in current silicon-based devices a transistor and capacitor are required to store a bit in dynamic RAM or four to six transistors are required to store a bit in static RAM. Second, the switching time for a $20-\mathrm{nm}$ device, $10^{-}$ ${ }^{11} \mathrm{~s}$, suggests that ON/OFF switching operations can be carried out at $100 \mathrm{GHz}$ (22). This switching time will decrease to $\sim 5 \times 10^{-12} \mathrm{~s}$ (200-GHz operation frequency) for a 5-nm element owing to the smaller effective mass, because the switching time is determined by the time to move the upper nanotube between its $\mathrm{ON}$ or OFF positions. The electrostatic charging time could also be important in device arrays made from SWNTs longer than 10 to $100 \mu \mathrm{m}$ (22). Finally, we note that the nonvolatile nature of our devices is preferable from the standpoint of power consumption and corresponding heat dissipation as compared to dynamic RAM, which must be continually refreshed.
To determine whether this nanotube device concept can be realized, we have studied the properties of suspended, crossed nanotube devices made from SWNT ropes (23) by mechanical manipulation (Figure 4). Current-voltage $(I-V)$ measurements made on the lower and upper nanotubes of a typical model device show ohmic behavior with resistances of 11 and 58 kilohms, respectively (Figure 4A). The $I-V$ curves between the upper and lower ropes in the OFF state were nonlinear, which is consistent with tunneling, with a resistance on the order of a gigohm. After switching ON, the $I-V$ curves exhibited ohmic behavior with a resistance of 112 kilohms (Figure 4B). This large change in resistance is consistent with our predictions for OFF versus ON states in the suspended device architecture. Reversible switching between well-defined ON and OFF states has also been observed in several devices (Figure $4 \mathrm{C}$ ). The smaller change in ON/OFF resistances for the device in Figure 4C is thought to arise from large contact resistances that are sometimes observed with nanotube ropes (24). Nevertheless, this change between $\mathrm{ON}$ and OFF states is 10-fold and persisted over several days of study. We think that these experiments represent clear proof of concept for our proposed architecture. Lastly, we have found that some of the devices fabricated from ropes could only be switched ON for reasonable applied voltages. This behavior is expected for potentials that have deep vdW minima (Figure 2A). Irreversible switching could be exploited to configure logic elements for computing (3).

We think that our calculations and experimental results clearly demonstrate the potential of our nanotube-based device architecture. There are several issues that must be addressed in order to take the next steps toward integrated molecular electronics. First, it is recognized that current SWNT samples consist of a random distribution of metallic (M) and semiconducting (S) tubes (8), and this might complicate device reading. However, the differences in resistance for $\mathrm{M} / \mathrm{M}, \mathrm{S} /$ $\mathrm{S}$, and M/S SWNT crosses are much smaller than that between the ON and OFF states, and thus, these states will be robust even with a mixture of different tubes. Second, an inherent limitation of crossbar memory architectures, such as in Figure 1, is the possibility of multiple electrical pathways (25). A standard solution to this problem is the incorporation of diodes at each cross point. This effective solution could be implemented in our system without the incorporation of additional elements by using semiconductor nanotubes or nanowires (7) for the lower molecular wires and metallic nanotubes for the upper SWNT because this would create a rectifying M/S junction at each cross point. Although it is not clear that it will be possible to exploit such an elegant solution with nanotubes until separated $\mathrm{M}$ and S SWNTs can be produced, the use of semiconductor nanowires for the lower 

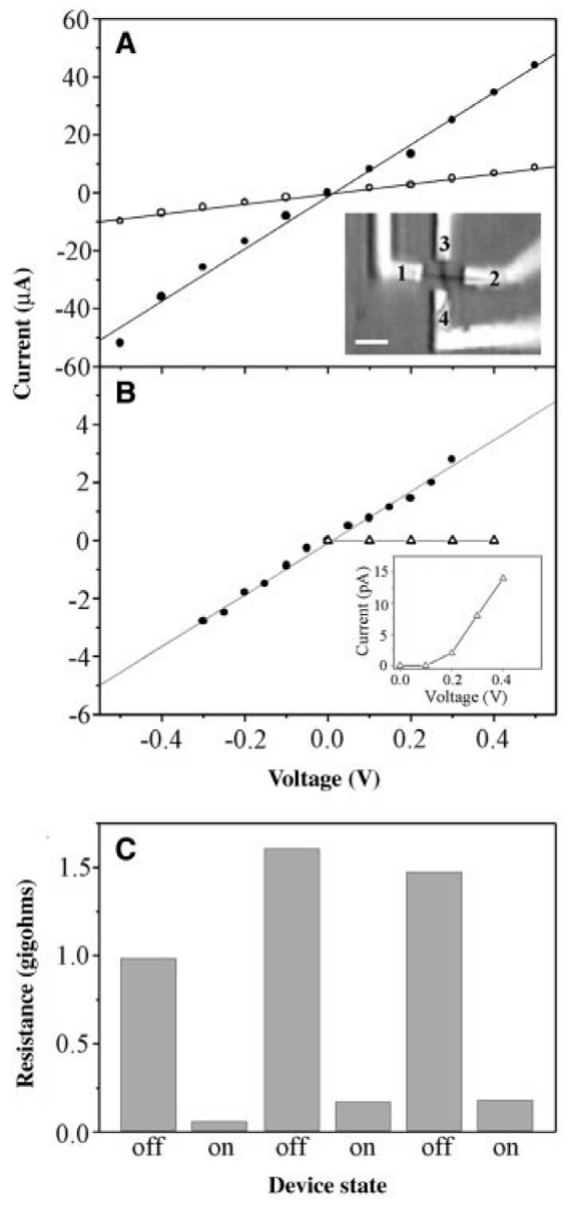

Figure 4. Carbon nanotube devices. (A) $I-V$ curves for the lower (electrodes 1 and 2, solid circles) and upper nanotubes (electrodes 3 and 4 , open circles). The two-terminal resistances are 11 and 58 kilohms, respectively. The inset shows a dark-field optical micrograph of the nanotube crossing (scale bar, $4 \mu \mathrm{m}$ ). The 150-nm-high chromium-gold electrodes were fabricated using electron beam lithography on an oxidized (600-nm-thick $\mathrm{SiO}_{2}$ ) highly doped silicon substrate. The gap between electrodes 1 and 2 is $5 \mu \mathrm{m}$, and that between electrodes 3 and 4 is $3.4 \mu \mathrm{m}$. Fiftynanometer-thick nanotube ropes were attached to the electrodes from nanotube-platinum tips using a micromanipulator under an optical microscope. (B) $I-V$ curves of the nanotube junction in OFF (open triangles) and ON (solid circles) states measured between electrodes 2 and 3; the junction was switched from OFF to ON at $2.5 \mathrm{~V}$. The ON resistance, 112 kilohms, is $\sim 10^{5}$ times lower than the OFF resistance. The inset shows the OFF state $I-$ $V$ on an expanded current scale. (C) Reversible switching of a suspended nanotube junction similar to the one shown in the inset of (A). The bias voltage for switching the junction ON was $\pm 5 \mathrm{~V}$ and for switching OFF was $40 \mathrm{~V}$. The resistance ratio between OFF and ON states is $R_{\text {off }} / R_{\text {on }} \sim 10$, with average OFF and ON resistances of $R_{\text {off }}=1.36$ gigohms and $R_{\text {on }}=140$ megohms, respectively. The junction was reversibly switchable for several days. All of the $I-V$ data were recorded under ambient conditions at room temperature. wires could lead to rectification at each cross, independent of the type of suspended SWNT (26). Lastly, it will ultimately be important to create devices in parallel using individual SWNTs. We think that this will be possible using either direct metal-catalyzed chemical vapor deposition (27) from patterned catalysts or directed assembly of nanotubes from solution (28). The developments in these growth and assembly areas suggest that highly integrated SWNT device arrays, which represent the next step in our plans for molecular electronics, may be soon realized.

\section{REFERENCES \& NOTES}

1. The 1997 National Technology Roadmap for Semiconductors (SEMATECH, Austin, TX, 1997).

2. M. C. Petty, M. R. Bryce, D. Bloor, Eds., Introduction to Molecular Electronics (Oxford Univ. Press, New York, 1995).

3. J. R. Heath, P. J. Kuekes, G. S. Snider, R. S. Williams, Science 280, 1716 (1998).

4. C. P. Collier, et al., Science 285, 391 (1999).

5. J. Chen, M. A. Reed, A. M. Rawlett, J. M. Tour, Science 286, 1550 (1999).

6. C. Dekker, Phys. Today 52, 22 (May 1999).

7. J. Hu, T. W. Odom, C. M. Lieber, Acc. Chem. Res. 32, 435 (1999).

8. T. W. Odom, J. L. Huang, P. Kim, C. M. Lieber, Nature 391, 62 (1998).

9. S. J. Tans, et al., Nature 386, 474 (1997) ; M. Bockrath, et al., Science 275, 1922 (1997).

10. S. J. Tans, A. R. M. Verschueren, C. Dekker, Nature 393, 49 (1998).

11. L. Chico, V. H. Crespi, L. X. Benedict, S. G. Louie, M. L. Cohen, Phys. Rev. Lett. 76, 971 (1996).

12. Z. Yao, H. W. Ch. Postma, L. Balents and C. Dekker, Nature 402, 273 (1999).

13. J. P. Lu, Phys. Rev. Lett. 79, 1297 (1997).

14. T. Hertel, R. E. Walkup, Ph. Avouris, Phys. Rev. B 58, 13870 (1998).

15. The elastic energy of a nanotube suspended on hard and soft supports has been evaluated using linear (Equation 2) and nonlinear beam-bending models (K. Kim, E. Joselevich, T. Rueckes, J. W. Hutchinson, C. M. Lieber, manuscript in preparation). Calculations using the nonlinear model agree to within $1 \%$ of those using the computationally less costly linear model (Equation 2).

16. M. B. Nardelli, B. I. Yakobson, J. Bernholc, Phys. Rev. Lett. 81, 4656 (1998).

17. D. A. Walters, et al., Appl. Phys. Lett. 74, 3803 (1999).

18. Our recent studies of nanotube tweezers [ P. Kim and C. M. Lieber, Science 286, 2148 (1999)] show experimentally that nanotubes can behave as robust electromechanical devices. In this latter work, the free ends of nanotube bundles could be deflected repeatably using electrostatic forces to grab submicron objects.

19. The electrostatic energy of the system was evaluated by solving the Laplace equation for the suspended nanotube geometry (Figure 1), including the dielectric support layer. The Laplace equation in a general 3D geometry was solved using Green's theorem
[J. D. Jackson, Classical Electrodynamics (Wiley, New York, ed. 2, 1974)]. Green's theorem converts the Laplace equation into the form of an integral equation. It implies that if the potential and its normal derivative on the boundary are known, then the electrostatic potential over all 3D space can be determined. The value of the potential on the boundary is known, so the main task is to solve for the normal derivative. We used the potential value on the boundary to self-consistently obtain the normal derivative with the boundary element method [C. A. Brebbia, The Boundary Element Method for Engineers (Wiley, New York, 1978)].

20. Y. H. Lee, S. G. Kim, D. Tománek, Chem. Phys. Lett. 265, 667 (1997).

21. C. Joachim and J. K. Gimzewski, Chem. Phys. Lett. 265, 353 (1997).

22. The mechanical switching time, $T_{\text {mech }}$, was calculated by reducing the problem to that of an effective particle moving along the potential energy surface. At the switching voltage (where there is no barrier), $T_{\text {mech }}=\int d z(\mu / 2[E-V(z)])^{1 / 2}$, where $\mu$ is the effective mass of the suspended $(10,10)$ SWNT, $E$ is the initial energy, and $V(z)$ is the switching potential. The resistance $\cdot \mathrm{ca}$ pacitance $(R C)$ time constant is calculated by assuming long SWNTs, which could form highly integrated structures containing on the order of $10^{6}$ device elements (that is, $\sim 10-\mu \mathrm{m}$ tubes). The capacitance, $C$, is given by $C=2 \pi \varepsilon L / \log (2 h / r)$, where $\varepsilon$ is the dielectric constant of the dielectric layer, $L$ is the nanotube length, $r$ is the nanotube radius, and $h$ is the height of the dielectric layer. The calculated nanotube capacitance of $10^{-4} \mathrm{pF}$ for a $10-\mu \mathrm{m}$ tube leads to an $R C$ limited operation frequency on the order of $100 \mathrm{GHz}$. The capacitance of the nanotube-nanotube tunnel junction is negligibly small $\left(10^{-7} \mathrm{pF}\right)$ so that it will not affect the speed of the device.

23. A. Thess, et al., Science 273, 483 (1996).

24. A. Bachtold, et al., Appl. Phys. Lett. 73, 274 (1999).

25. G. A. Prinz, Science 282, 1660 (1998).

26. Semiconductor nanowires can be specifically doped n-type or p-type [ Y. Cui, X. Duan, J. Hu, C. M. Lieber, J. Phys. Chem. $B$ 104, 5213 (2000)]. If the lower wires in our architecture are made from n-type nanowires, then rectifying behavior will be observed at each cross element, irrespective of whether the upper SWNT is metallic or semiconductor. Metallic SWNTs would produce M-S diode junctions, whereas semiconducting SWNTs, which (as previous studies have shown) behave as p-type materials (12), would form rectifying p-n junctions.

27. J. Kong, H. T. Soh, A. M. Cassell, C. F. Quate, H. Dai, Nature 395, 878 (1998).

28. J. Liu, et al., Chem. Phys. Lett. 303, 125 (1999).

29. A. R. Burns, J. E. Houston, R. W. Carpick, T. A. Michalske, Langmuir 15, 2922 (1999).

30. We thank J. W. Hutchinson, P. Kim, and J. Huang for helpful discussion and E. J. Sánchez for help with figures. C.M.L. acknowledges support of this work by the Defense Advanced Research Projects Agency and the Office of Naval Research. 